\title{
Characterization of the Casein/Keratin Self-Assembly Nanomicelles
}

\author{
Su Xiao-Zhou, Wang Hong-Ru, and Huang Mian \\ College of Resources and Environment, Shaanxi University of Science and Technology, Xian 710021, China \\ Correspondence should be addressed to Wang Hong-Ru; wanghr@sust.edu.cn
}

Received 12 September 2013; Revised 16 January 2014; Accepted 19 January 2014; Published 25 February 2014

Academic Editor: Anchal Srivastava

Copyright (c) 2014 Su Xiao-Zhou et al. This is an open access article distributed under the Creative Commons Attribution License, which permits unrestricted use, distribution, and reproduction in any medium, provided the original work is properly cited.

\begin{abstract}
Complex nanomicelles were made from casein and keratin through electrostatic self-assembly and transglutaminase fixation that was proved to be harmless and green. The complex nanomicelles were characterized by dynamic light scattering, scanning electron microscopy, atomic force microscopy, Fourier transform infrared spectroscopy, differential scanning calorimetry, and steady-state florescence. The results show that the complex nanomcelles acquired at the neutral $\mathrm{pH}$ in the mass ratio of casein to keratin $4: 1$ exhibit an anomalous sphere shape with uniform size which the diameter is about $40-70 \mathrm{~nm}$. The complex nanomicelles in solution possess excellent dilution and storage stability due to the fixation and their high $\zeta$-potential $(22.8 \mathrm{mV})$. The complex nanomicelles are relatively hydrophilic and have a good potential for industrial application.
\end{abstract}

\section{Introduction}

Molecular self-assembly is the spontaneous association of molecules under equilibrium conditions into stable, structurally well-defined aggregates joined by noncovalent bonds [1]. Self-assembly of biomacromolecules is emerging as a new route to produce novel materials, which can find many applications in biomedical technology and biomaterials technology.

Both casein and keratin have a strong tendency to selfassemble spontaneously into micelles in solution and their spontaneous self-assembly has been studied extensively in recent years [2-7]. Casein has an isoelectric point of 4.6, and it is relatively hydrophobic, making it poorly soluble in water. High glycine-tyrosine keratin from wool has an isoelectric point around 6.5, and it is relatively hydrophilic in water $[8,9]$. When these different types of proteins were mixed together, they did not result in precipitation and phase separation. Therefore, the hydrophobic effect and the electrostatic interaction can be employed to reassemble and fabricate the casein/keratin nanomicelles.

The polymer micelles were characterized by their good drug loading capacity, high structural stability, excellent water solubility, and tiny particle size $(100 \mathrm{~nm}$ or smaller).
Based on these viewpoints, our studies intended for structural stability and nanoparticles have been conducted. Biomaterials used for drug delivery, ecological coating, or cosmetic ingredients with desired properties will be developed based on the complex casein/keratin nanomicelles without toxicity.

\section{Materials and Methods}

2.1. Materials. Casein (technical grade) was purchased from Sigma Company Ltd. High glycine-tyrosine keratin powder was prepared from wool hydrolysate by stepwise precipitation with acid. All the other reagents used were of analytical grade.

2.2. Preparation of the Complex Nanomicelles. Casein was dispersed in ultrapure water by magnetic stirring at $50^{\circ} \mathrm{C}$ and then stored at $4^{\circ} \mathrm{C}$ at least for 10 days. The casein solution $(5 \mathrm{mg} / \mathrm{mL})$ was prepared and adjusted to $\mathrm{pH} 7.0$ with $1 \mathrm{M}$ $\mathrm{NaOH}$ and $1 \mathrm{M} \mathrm{HCl}$ solutions. High glycine-tyrosine keratin powder was put into ultrapure water and solid $\mathrm{NaOH}$ was added until the keratin completely dissolved by magnetic stirring. The casein and keratin solutions were then filtrated by millipore filters with $2.0 \mu \mathrm{m}$ pore size to get rid of the impurities and microbes. Then the casein and keratin 
TABLE 1: Influence of the mass ratio of casein to keratin on the $D_{h}$, PDI, and scattering light intensity of the casein/keratin complex nanomicelles.

\begin{tabular}{lccc}
\hline $\mathrm{C} / \mathrm{K}$ & $D_{h}(\mathrm{~nm})$ & PDI & Intensity (counts) \\
\hline $1: 0$ & $70.85 \pm 1.6$ & $0.591 \pm 0.04$ & $82.47 \pm 12$ \\
$8: 1$ & $65.22 \pm 1.4$ & $0.522 \pm 0.03$ & $72.26 \pm 9$ \\
$4: 1$ & $60.24 \pm 1.1$ & $0.425 \pm 0.01$ & $96.53 \pm 18$ \\
$2: 1$ & $64.55 \pm 1.8$ & $0.613 \pm 0.06$ & $92.31 \pm 11$ \\
$1: 1$ & $50.48 \pm 1.5$ & $0.625 \pm 0.09$ & $66.89 \pm 7$ \\
$1: 2$ & $46.57 \pm 0.8$ & $0.611 \pm 0.08$ & $75.25 \pm 14$ \\
$1: 4$ & $42.15 \pm 0.6$ & $0.654 \pm 0.07$ & $89.95 \pm 12$ \\
$1: 8$ & $48.58 \pm 0.8$ & $0.687 \pm 0.09$ & $63.25 \pm 12$ \\
$0: 1$ & $120.23 \pm 2.8$ & $0.712 \pm 0.08$ & $60.54 \pm 10$ \\
\hline
\end{tabular}

Note: $\mathrm{C} / \mathrm{K}$ represents the casein/keratin nanomicelles. $D_{h}$ is the hydrodynamic diameters; PDI is the particle dispersion index.

solutions were dispersed with sodium citrate in the mass ratio of protein to sodium citrate $5: 1$. Casein and keratin solutions were then mixed gently under different conditions and kept at $4^{\circ} \mathrm{C}$ for at least 24 hours to allow the casein and keratin to self-assemble completely. The complex micelles were then fixed by transglutaminase for 1 hour at $50^{\circ} \mathrm{C}$ and the enzyme was inactivated at $80^{\circ} \mathrm{C}$ for $10 \mathrm{~min}$.

2.3. Turbidity Measurements. A Shimadzu UV 1705 spectrometer was used for turbidity measurements. Solution was injected into the Quartz cuvettes and the turbidity was detected at $600 \mathrm{~nm}$ wavelength.

2.4. Dynamic Light Scattering (DLS) Measurements. Hydrodynamic diameter of the micelles was measured by a Zeta Sizer Nano ZS90 (Malvern Instrument, Worcs, UK) equipped with $4 \mathrm{~mW}$ He-Ne Laser. The measurements were performed at a scattering angle of $90^{\circ}$ at $25.0 \pm 0.1^{\circ} \mathrm{C}$. The apparent $z$ average diameter $\left(D_{h}\right)$, polydispersity index (PDI), and the intensity for $z$-average were calculated by the Dispersion Technology Software provided by Malvern. The concentration of the micelles for DLS measurements was $5 \mathrm{mg} / \mathrm{mL}$ in each sample.

2.5. $\zeta$-Potential Measurements. The $\zeta$-potential was measured on a Zeta Sizer Nano ZS90 (Malvern Instrument, Worcs, $\mathrm{UK})$ by using laser doppler microelectrophoresis. In this technique, an electric field was applied to the micelles, which then moved with a velocity related to their $\zeta$-potential. This velocity was measured using a patented laser interferometric technique called M3-PALS (phase analysis light scattering). This enabled the calculation of electrophoretic mobility. The test was carried out at $25.0 \pm 0.1^{\circ} \mathrm{C}$. The electrophoresis mobility $\left(U_{E}\right)$ was used to calculate the potential $(\zeta)$ by the Henry equation $U_{E}=2 \varepsilon \zeta f(\mathrm{ka}) / 3 \eta$, and $\varepsilon, \eta$, and $f(\mathrm{ka})$ represented, respectively, the dielectric constant, the viscosity of the medium, and Henry's function.

2.6. Scanning Electron Microscopy (SEM) Measurements. Hitachi S-4800 field emission scanning electron microscope was used to observe the micelles at $1 \mathrm{kV}$. The micelles were fixed by $2 \%$ glutaraldehyde on the silicon surface for at least $30 \mathrm{~min}$ in order to keep the micelles primitive form, then washed with deionized water, and dried at the room temperature. Before observation, the specimens were coated with gold.

2.7. Atomic Force Microscopy (AFM) Measurements. AFM samples were prepared by dropping the solution on freshly cleaved mica surface, and then the micelles were fixed by $2 \%$ glutaraldehyde for at least $30 \mathrm{~min}$. The samples were washed with deionized water and dried at room temperature. Skiko Atomic force Microscopy SPA400-SPI3800N was used to analyze the micelles in tapping mode.

2.8. Fourier Transform Infrared Spectroscopy Measurements. FT-IR spectra of the complex nanomicelles and the complex nanomicelles fixed by transglutaminase were measured with an Equinox 55 Spectrometer (Bruker). The analysis was performed in triplicate.

2.9. Thermal Analysis. Differential scanning calorimetry (DSC) measurements were performed with an instrument (netzsch STA 409) from room temperature to $400^{\circ} \mathrm{C}$ at a heating rate of $10^{\circ} \mathrm{C} / \mathrm{min}$. The complex nanomicelles and the complex nanomicelles fixed by transglutaminase were kept at $105^{\circ} \mathrm{C}$ for $30 \mathrm{~min}$ to get rid of the moisture.

2.10. Steady-State Fluorescence Measurements. The steadystate fluorescence tests were carried out on a fluorescence spectrophotometer Hitachi F-7000. Recrystallized pyrene was dissolved in acetone to prepare a concentration of $2 \times 10^{-5} \mathrm{~g} / \mathrm{mL}$ stock solution and its final concentration in micelles solution was $2 \times 10^{-7} \mathrm{~g} / \mathrm{mL}$ for test. Before measurements, the micelles solution was stored at $4^{\circ} \mathrm{C}$ for 24 hours after the pyrene was added. The excitation and emission wavelength were recorded at the $338 \mathrm{~nm}, 381 \mathrm{~nm}$, and $373 \mathrm{~nm}$ wavelengths.

\section{Results and Discussion}

3.1. Diameter of the Complex Nanomicelles in Solution. The influence of mass ratio of casein to keratin on complex nanomicelles diameter was investigated at neutral $\mathrm{pH}$. The DLS measurements revealed that the two proteins interacted with each other and formed complex micelles with diameter about $40-65 \mathrm{~nm}$ at $\mathrm{pH} 7.0$ (Table 1). It was also found that any of the diameters of the complex nanomicelles in different mass ratio of casein and keratin were smaller than that of either casein micelles or keratin particles. This proved that the two protein particles had a strong hydrophobicity in the reassembly process. The casein micelles and keratin particles dissociated partly when these two proteins mixed together. The keratin peptides competed with casein peptides and bound to the interior sites of the casein micelles and led to the dissociation of the colloidal calcium phosphate (CCP). Table 1 also showed that the diameters of the complex nanomicelles decreased when the keratin content increased, which implied 


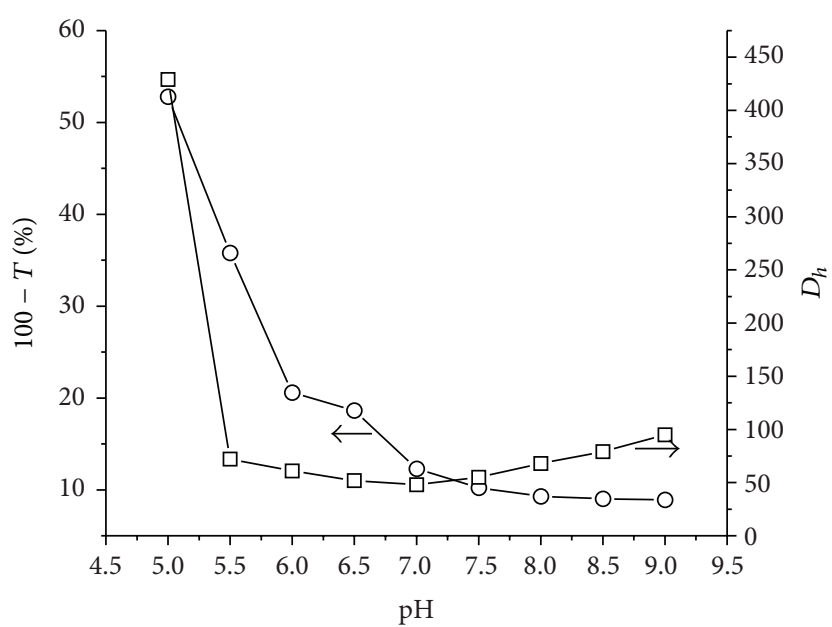

FIGURE 1: Turbidity and diameters of the casein/keratin complex nanomicelles $(5 \mathrm{mg} / \mathrm{mL})$ as a result of $\mathrm{pH}$ value in the mass ratio of casein to keratin $4: 1$.

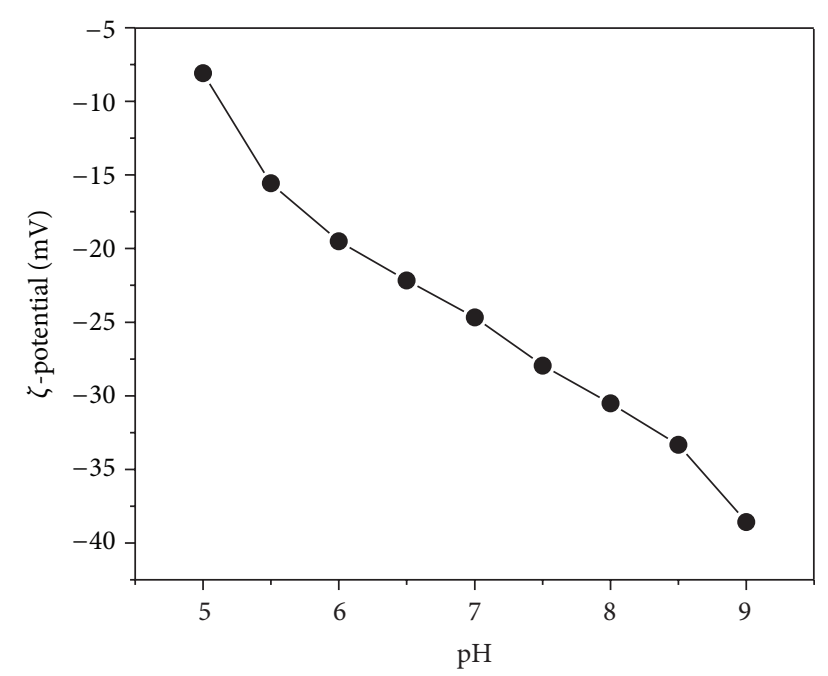

FIGURE 2: $\zeta$-potential of the casein/keratin complex nanomicelles. The micelles were prepared in the mass ratio of casein to keratin $4: 1$ and at $\mathrm{pH}$ 5.0-9.0.

that the keratin particles played a major role in nanomicelle dispersion process.

The data in Table 1 also showed that the nanomicelles prepared at neutral $\mathrm{pH}$ in mass ratio of casein to keratin $4: 1 \mathrm{had}$ the highest intensity and relatively narrow PDI. Therefore, the smallest and stable nanomicelles can be obtained at the neutral $\mathrm{pH}$ in the mass ratio of casein to keratin $4: 1$, of which the average diameter is $60 \mathrm{~nm}$ (Figure 1).

3.2. $\zeta$-Potential of the Complex Nanomicelles. $\zeta$-potential relates to the net charges on the surface of the particles in solution. Figure 2 showed that the absolute value of the $\zeta$ potential of the complex nanomicelles increased with the enhancement of $\mathrm{pH}$ value from 5.0 to 9.0 in the mass ratio of 4: 1 . This illustrated that the complex nanomicelles became

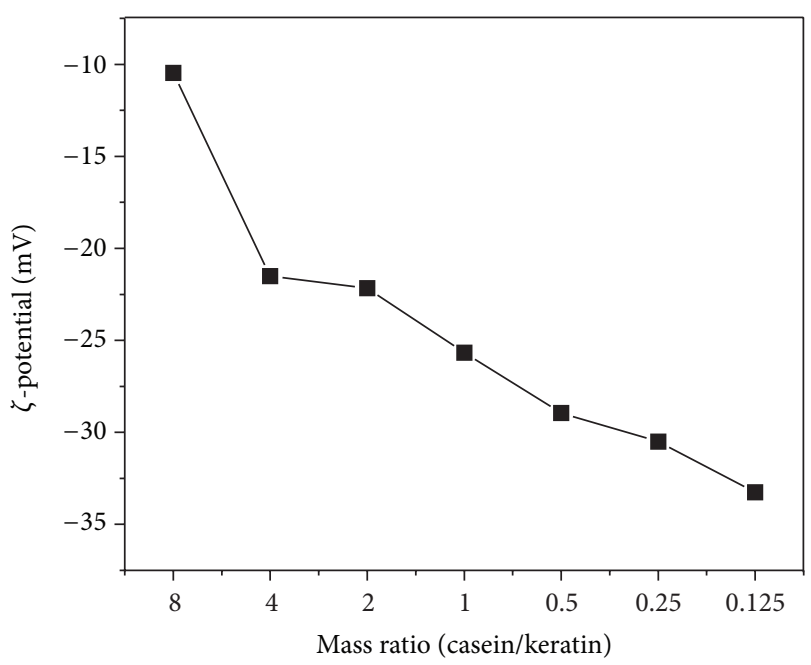

FIGURE 3: $\zeta$-potential of the casein/keratin complex nanomicelles prepared at $\mathrm{pH} 7.0$ in different mass ratios.

stabilized when the solution $\mathrm{pH}$ value increased from 5.0 to 9.0.

Figure 3 showed the $\zeta$-potential of the complex micelles in different mass ratios of casein and keratin at neutral $\mathrm{pH}$. The $\zeta$-potential absolute value of the complex micelles increased with the increase of the keratin content. It can be inferred that the introduction of the keratin increased the stability of the complex nanomicelles.

3.3. Morphology of the Complex Nanomicelles. The morphology of the casein/keratin complex nanomicelles was observed by SEM. The samples in Figures 4(a) and 4(b) were from the 100 -fold diluted self-assembly solution, and the samples in Figures 4(c) and 4(d) were from the 200-fold diluted selfassembly solution.

Figures 4(a) and 4(c) represented the nanomicelles' image, and Figures 4(b) and 4(d) represented the nanomicelles' image fixed by transglutaminase. The complex nanomicelles made from casein and keratin via self-assembly had a diameter of about 20-40 nm (Figures 4(a) and 4(c)). The fixed complex nanomicelles had a diameter of $40-70 \mathrm{~nm}$ (Figures 4(b) and 4(d)). The fixation with transglutaminase led to the enhancement of the micelles' diameter. In addition, two different fold-diluted samples showed almost the same diameter and morphology of the micelles (Figures 4(b) and $4(d)$ ), which indicated that the dilution did not result in the dissociation of the complex nanomicelles, and the complex nanomicelles were stable on dilution. These implied that the crosslinking was formed either in the nanomicelles or between the nanomicelles during the fixation with transglutaminase.

Figure 5 showed the topography image of the fixed complex nanomicelles by the atomic force microscope. The morphology of the nanomicelles was of nearly spherical shape and had a diameter of $40-70 \mathrm{~nm}$. This result was in accordance with the result measured by SEM and DLS. The diameter of the nanomicelles measured in solid state (SEM, 


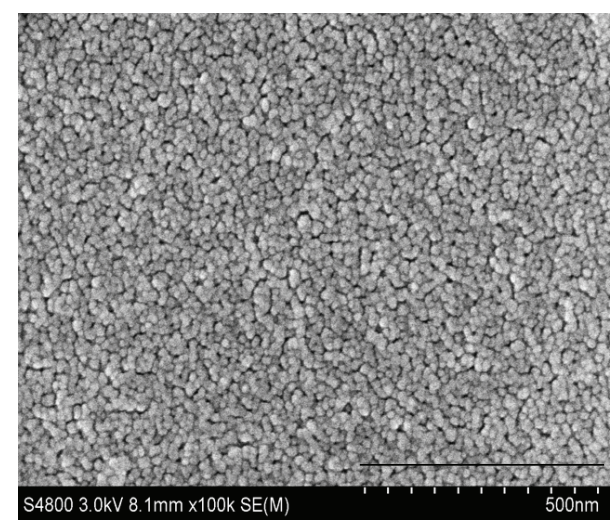

(a)

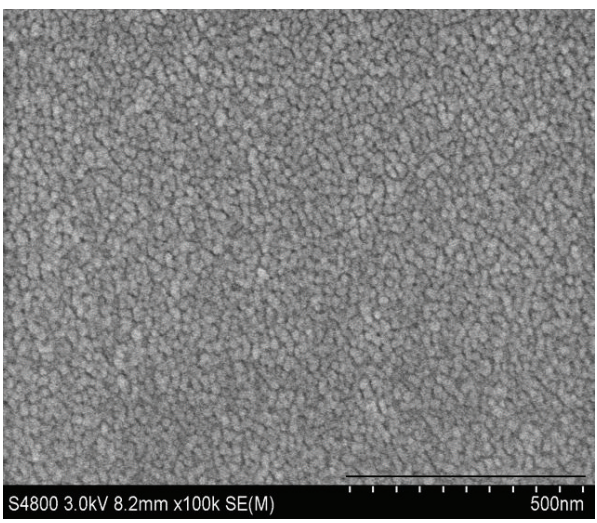

(c)

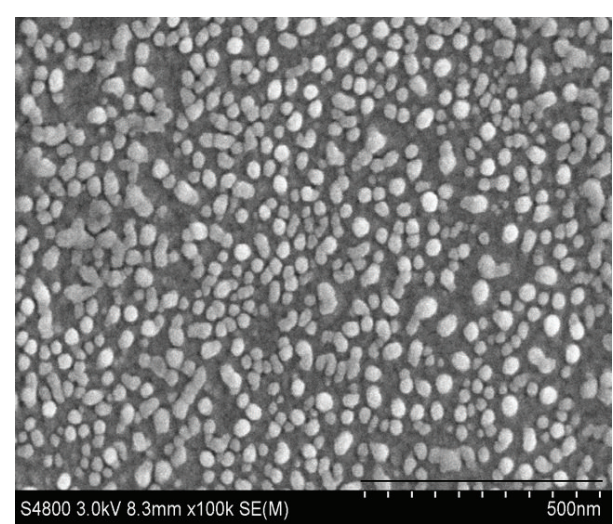

(b)

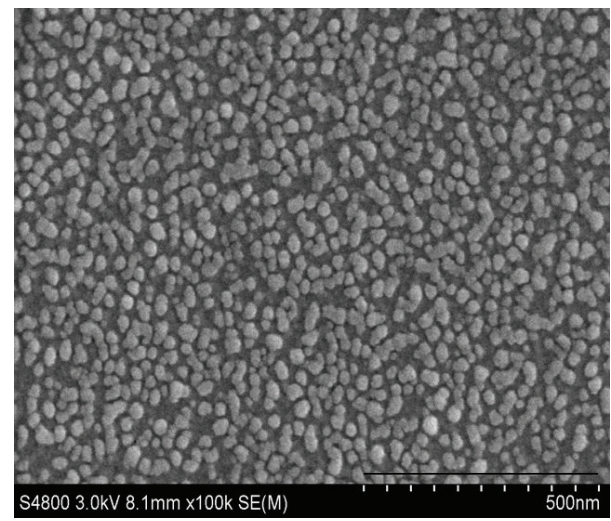

(d)

FIGURE 4: SEM images of the casein/keratin complex nanomicelles. (a) and (b) were diluted by 100-fold. (c) and (d) were diluted by 200fold. (a) and (c) were casein/keratin complex nanomicelles; (b) and (d) were complex nanomicelles treated with transglutaminase (100 k magnification).

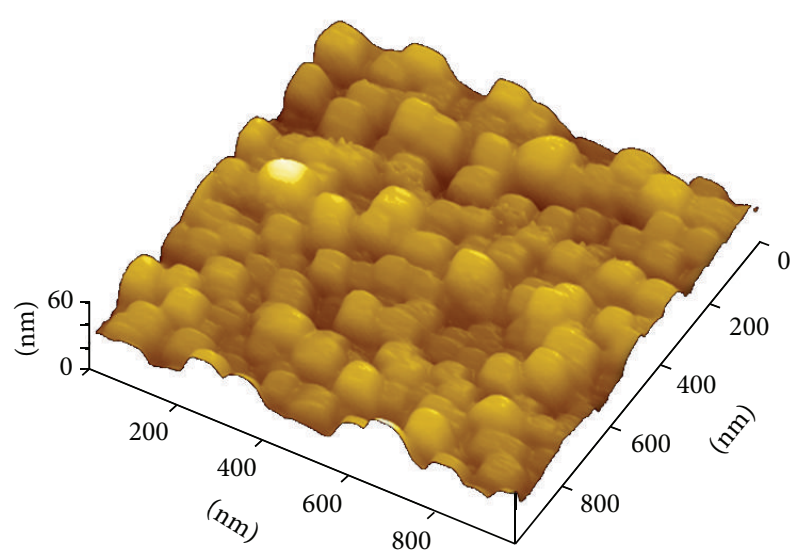

Figure 5: AFM images of the fixed casein/keratin complex micelles.

AFM) and in solution (DLS) had no great difference. This is attributed to the unique structure of the nanomicelles and the fixation method.

The FTIR spectrum of the complex nanomicelles showed an absorption band at $1113 \mathrm{~cm}^{-1}$ (Figure 6, CK-), but the complex nanomicelles fixed with transglutaminase showed

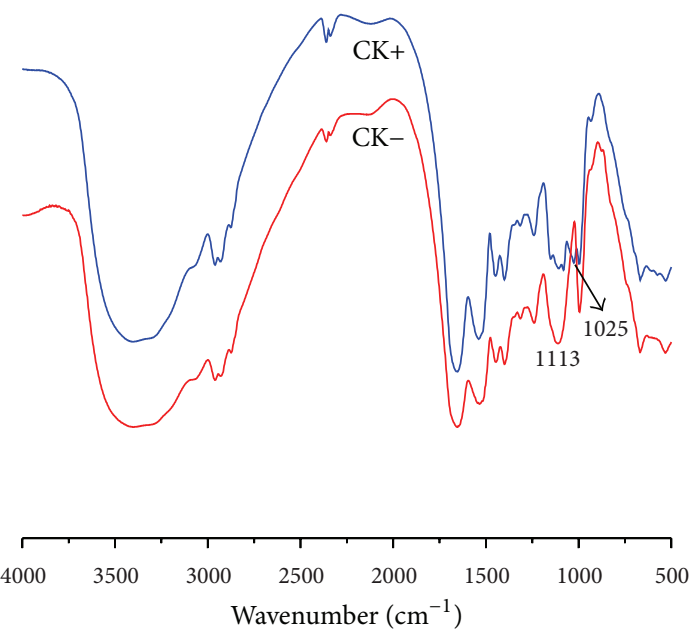

FIGURE 6: FT-IR spectra of the casein/keratin nanomicelles mixtures $(\mathrm{CK}-)$ and mixture treated with transglutaminase $(\mathrm{CK}+)$.

three absorption bands, respectively, at $1150 \mathrm{~cm}^{-1}, 1107 \mathrm{~cm}^{-1}$, and $1081 \mathrm{~cm}^{-1}$ in the corresponding area (Figure 6, CK+). The absorption in this area is related to the $\mathrm{C}-\mathrm{N}$ stretching 


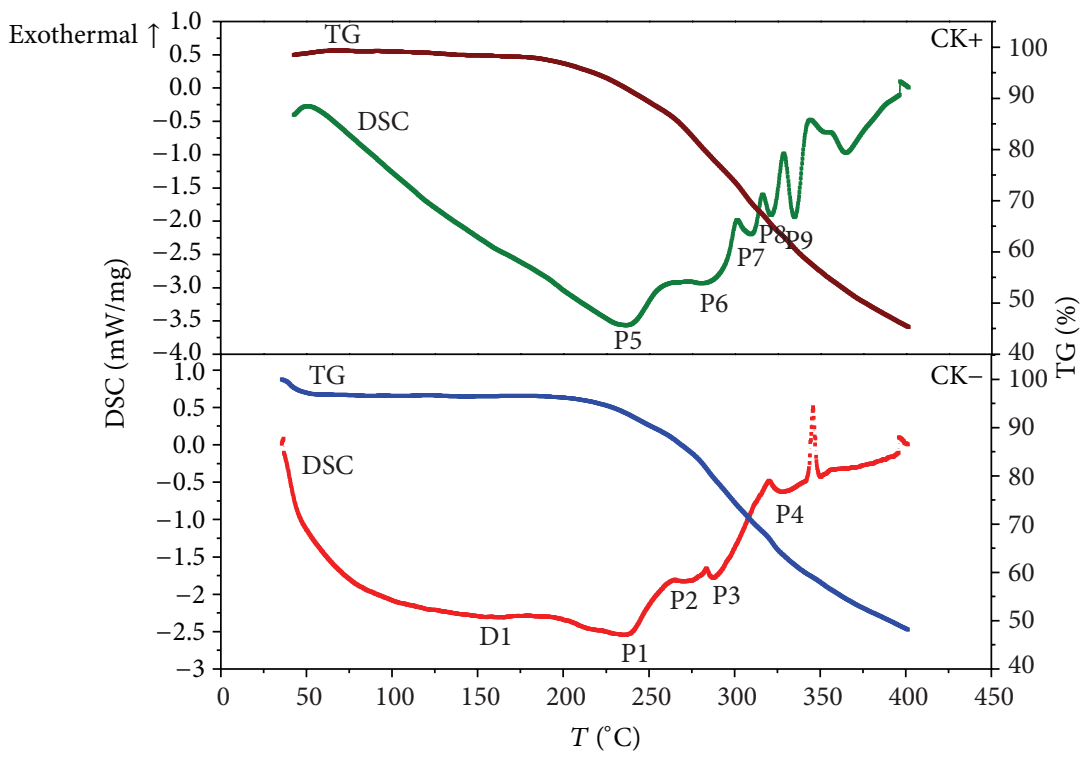

FIGURE 7: DSC thermograms of the casein/keratin nanomicelles mixture (CK-) and mixture treated by transglutaminase (CK+).

vibration of amido bonds. This indicated that some different new amido bonds were formed during the fixation with transglutaminase. Furthermore, the complex nanomicelles fixed with transglutaminase exhibited an additional band at $1025 \mathrm{~cm}^{-1}$ (Figure 6, CK+). On the basis of the transglutaminase spectrum (spectrum not shown) it was determined that this band came from the saccharide structure of malt dextrin incorporated into preparation of commercial transglutaminase.

3.4. Thermal Performance of the Complex Nanomicelles. The TG curve and the DSC curve of the complex nanomicelles were shown in Figure 7 ( $\mathrm{CK}-$ ). The wide heat absorption band $\mathrm{D} 1\left(120-180^{\circ} \mathrm{C}\right)$ was related to the phase transition of the hydrated casein/keratin crystal. Peak $1\left(238^{\circ} \mathrm{C}\right)$ corresponded to the cleavage of casein/keratin crystal. Peak $2\left(275^{\circ} \mathrm{C}\right)$ corresponded to the breakdown of crosslinks (-s-s- bonds, $\mathrm{H}$ bonds, and salt links) in keratin. Peak $3\left(290^{\circ} \mathrm{C}\right)$ corresponded to the rupture of peptide bonds of casein. Peak $4\left(330^{\circ} \mathrm{C}\right)$ corresponded to the liquefaction and rupture of peptide bond of keratin $[10,11]$.

The TG curve and the DSC curve of the complex nanomicelles fixed with transglutaminase were shown in Figure 7 $(\mathrm{CK}+)$. In Figure $7(\mathrm{CK}+)$, the wide heat absorption band $\left(120-180^{\circ} \mathrm{C}\right)$ related to the phase transition of the hydrated casein/keratin crystal disappeared, which indicated that the hydration ability of casein/keratin micelles was reduced by the fixation with transglutaminase. The heat absorption related to the breakdown of crosslinks in keratin and peptide bonds in casein appeared at $283^{\circ} \mathrm{C}$ (peak 6) as a single band, which implied that either the crosslinks or the peptide bonds were altered by the fixation with transglutaminase. The cleavage of casein/keratin crystal was scarcely influenced by the fixation (peak 1 , peak 5$)$. Peak $7\left(310^{\circ} \mathrm{C}\right)$, peak 8 $\left(320^{\circ} \mathrm{C}\right)$, and peak $9\left(333^{\circ} \mathrm{C}\right)$ corresponded to the rupture of keratin peptide bonds from different components caused by the fixation with transglutaminase.

3.5. Hydrophobicity/Hydrophilicity of the Complex Nanomicelles. Recrystallized pyrene was used as a probe to detect the hydrophobicity and hydrophilicity of casein/keratin complex nanomicelles. The intensity ratio of the first to the third peak $\left(I_{1} / I_{3}\right)$ in the fluorescence spectrum can reflect the microenvironmental polarity where the probe exists [12]. The greater the value of $I_{1} / I_{3}$ was, the weaker the hydrophobic microenvironment for pyrene was.

Figure 8 showed that the value of $I_{1} / I_{3}$ for the complex nanomicelles was $\mathrm{pH}$ sensitive at $\mathrm{pH}$ 5.0-9.0. The value of $I_{1} / I_{3}$ of the complex nanomicelles increased with the increase of $\mathrm{pH}$ value from 5.0 to 9.0. The complex nanomicelle was more hydrophobic near the isoelectric point of the casein and keratin, which led to the relative lower value of $I_{1} / I_{3}$. When the $\mathrm{pH}$ was increased from 5.0 to 9.0, the micelles' structure became loose because of the increase in electrostatic repulsion and the more hydrophilic group stretched out from the micelles, which led to the relative higher value of $I_{1} / I_{3}$.

Figure 9 showed that the value of $I_{1} / I_{3}$ for the complex nanomicelles varied as a function of mass ratio. The value of $I_{1} / I_{3}$ of the micelles increased when the value of mass ratio of casein/keratin decreased. When the keratin was added gradually, the micelles disassociated partly and the structure became loose, and the micelles got more hydrophilic relatively.

3.6. Storage Stability of the Complex Nanomicelles. Casein nanomicelles $(5 \mathrm{mg} / \mathrm{mL})$ and casein/keratin complex nanomicelles $(5 \mathrm{mg} / \mathrm{mL}$, mass ratio $4: 1)$ at neutral $\mathrm{pH}$ were prepared separately and their stability was compared during storage at room temperature. The changes in diameters and PDI were showed in Figures 10 and 11. Figure 10 showed 


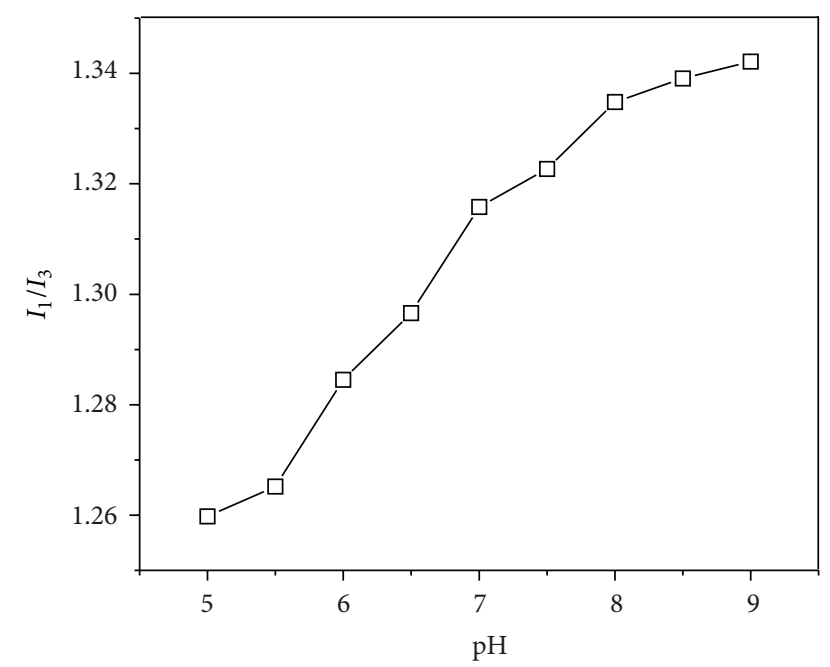

FIGURE 8: $I_{1} / I_{3}$ ratio of pyrene fluorescence for casein/keratin complex nanomicelles solution as functions of $\mathrm{pH}$ in the range of 5.0-9.0. The micelles solutions were prepared at $\mathrm{pH} 7.0$ with a protein concentration of $5 \mathrm{mg} / \mathrm{mL}$.

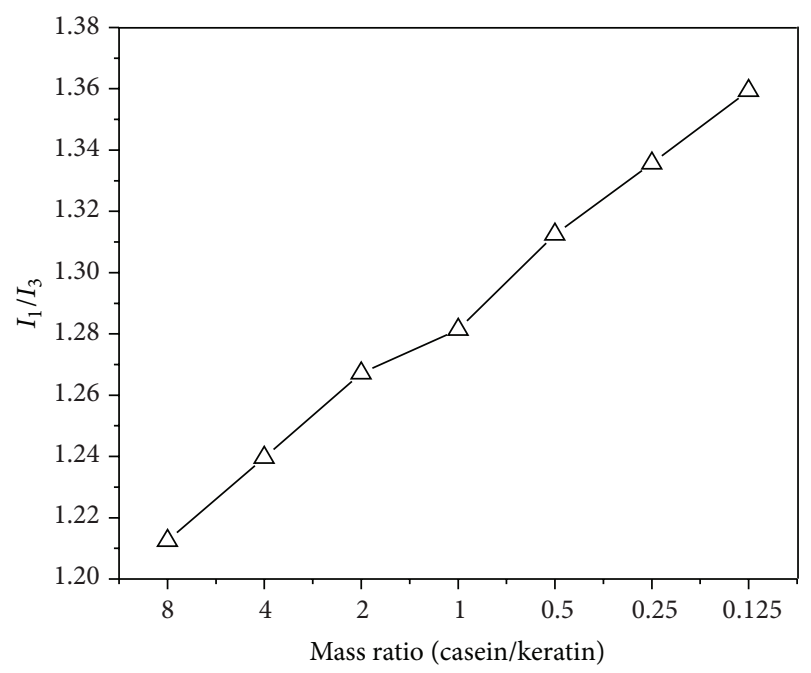

FIGURE 9: $I_{1} / I_{3}$ ratio of pyrene fluorescence for casein/keratin complex nanomicelles solution as functions of mass ratio. The micelles solutions were prepared at $\mathrm{pH} 7.0$ with a protein concentration of $5 \mathrm{mg} / \mathrm{mL}$.

that the value of the diameter for the complex nanomicelles almost was constant during the forty days of storage. On the contrary, casein micelles' diameter increased greatly in this process. The PDI value also exhibited similar result in Figure 11. These indicated that the nanomicelles' stability was improved dramatically when keratin was added. This is because the keratin employed possesses high stability in structure [13] and lower molecular weight with intact secondary structure.

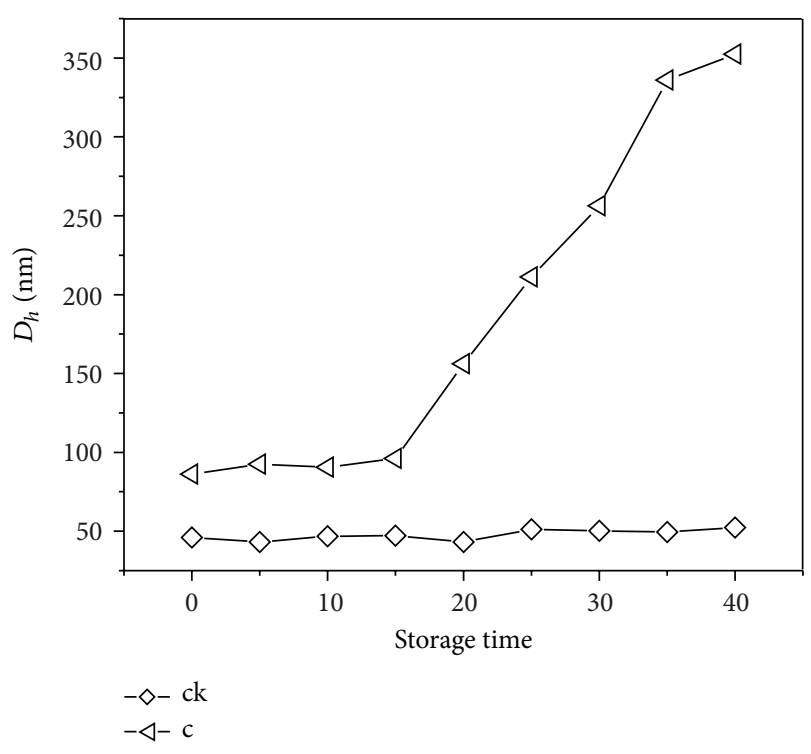

FIgURE 10: Plots of $D_{h}$ variations for the casein micelles and the casein/keratin complex nanomicelles in the 40-day preservation time. The micelles solutions were prepared at $\mathrm{pH} 7.0$ with a protein concentration of $5 \mathrm{mg} / \mathrm{mL}$.

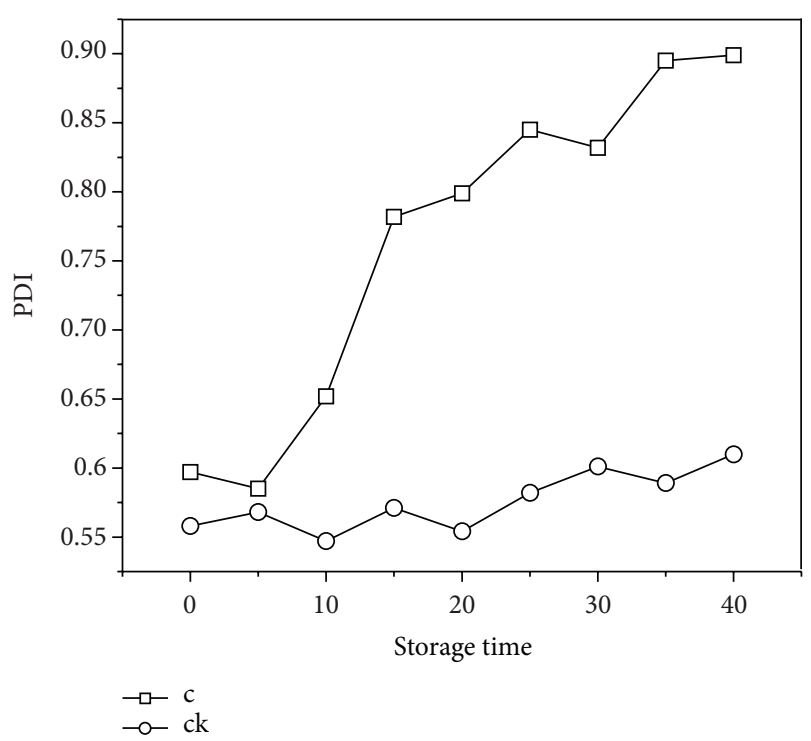

FIGURE 11: Plots of PDI variations for the casein micelles and the casein/keratin complex nanomicelles in the 40-day preservation time. The micelles solutions were prepared at $\mathrm{pH} 7.0$ with a protein concentration of $5 \mathrm{mg} / \mathrm{mL}$.

\section{Conclusions}

Casein and keratin were used to fabricate complex nanomicelles through electrostatic self-assembly. In this process, no harmful reagents were used. Polydisperse complex nanomicelles can be formed in $\mathrm{pH}$ range of 5.0-9.0. Stable complex nanomicelles can be acquired at the neutral $\mathrm{pH}$ in the mass ratio of casein to keratin $4: 1$, and the absolute value of $\zeta$-potential was $22.8 \mathrm{mV}$. The stable complex nanomicelles 
exhibited anomalous sphere shape with uniform size of which the average diameter was about $40-70 \mathrm{~nm}$. The fixation with transglutaminase could enhance the dilution stability and reduce the hydration of the complex nanomicelles to some extent by forming crosslinking in the nanomicelles or between the nanomicelles. The complex nanomicelles were relatively hydrophilic and had good storage stability.

\section{Conflict of Interests}

The authors declare that there is no conflict of interests regarding the publication of this paper.

\section{Acknowledgments}

This work was financially supported by the Hongliang Research Fund (413118) and Doctor Research Fund of Shaanxi University of Science and Technology (BJ08-15). The material in this work was supported by Jiangsu Yiming Bio-Products Co.

\section{References}

[1] G. M. Whitesides, J. P. Mathias, and C. T. Seto, "Molecular self-assembly and nanochemistry: a chemical strategy for the synthesis of nanostructures," Science, vol. 254, no. 5036, pp. 1312-1319, 1991.

[2] C. G. De Kruif, "Casein micelle interactions," International Dairy Journal, vol. 9, no. 3-6, pp. 183-188, 1999.

[3] C. F. Narambuena, F. S. Ausar, I. D. Bianco, D. M. Beltramo, and E. P. M. Leiva, "Aggregation of casein micelles by interactions with chitosans: a study by Monte Carlo simulations," Journal of Agricultural and Food Chemistry, vol. 53, no. 2, pp. 459-463, 2005.

[4] C. Garnier, C. Michon, S. Durand, G. Cuvelier, J.-L. Doublier, and B. Launay, "Iota-carrageenan/casein micelles interactions: evidence at different scales," Colloids and Surfaces B, vol. 31, no. 1-4, pp. 177-184, 2003.

[5] M. van de Locht, "Reconstitution of microfibrils from wool and filaments from epidermis proteins," Melliand Textilberichte, vol. 10, pp. 780-786, 1987.

[6] P. M. Steinert and M. I. Gullino, "Bovine epidermal keratin filament assembly in vitro," Biochemical and Biophysical Research Communications, vol. 70, no. 1, pp. 221-227, 1976.

[7] H. Thomas, A. Conrads, P. H. Phan, M. van de Locht, and H. Zahn, "In vitor reconstitution of wool intermediate filaments," International Journal of Biological Macromolecules, vol. 8, pp. 258-264, 1986.

[8] J. E. Plowman, “The proteomics of keratin proteins," Journal of Chromatography B, vol. 849, no. 1-2, pp. 181-189, 2007.

[9] L. M. Flanagan, J. E. Plowman, and W. G. Bryson, “The high sulphur proteins of wool: towards an understanding of sheep breed diversity," Proteomics, vol. 2, no. 9, pp. 1240-1246, 2002.

[10] W. Xu, W. Guo, and W. Li, "Thermal analysis of ultrafine wool powder," Journal of Applied Polymer Science, vol. 87, no. 14, pp. 2372-2376, 2003.

[11] B. Purevsuren and Y. Davaajav, "Thermal analysis of casein," Journal of Thermal Analysis and Calorimetry, vol. 65, no. 1, pp. 147-152, 2001.
[12] C. Keyes-Baig, J. Duhamel, S. Fung, and J. Bezaire, "Selfassembling peptide as a potential carrier of hydrophobic compounds," Journal of the American Chemical Society, vol. 126, no. 24, pp. 7522-7532, 2004.

[13] J. Li, Y. Li, L. Li, A. F. T. Mak, F. Ko, and L. Qin, "Preparation and biodegradation of electrospun PLLA/keratin nonwoven fibrous membrane," Polymer Degradation and Stability, vol. 94, no. 10, pp. 1800-1807, 2009. 

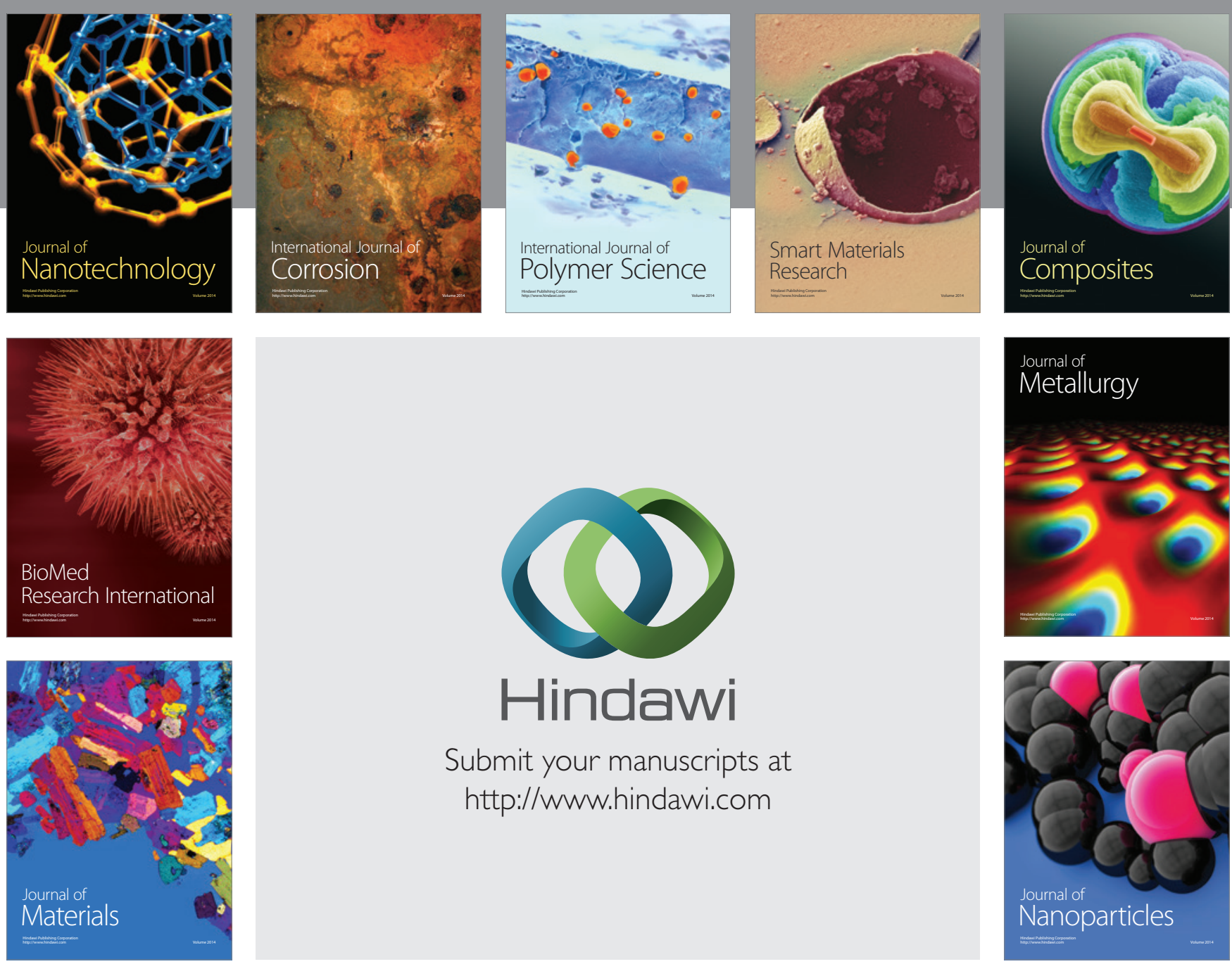

Submit your manuscripts at http://www.hindawi.com
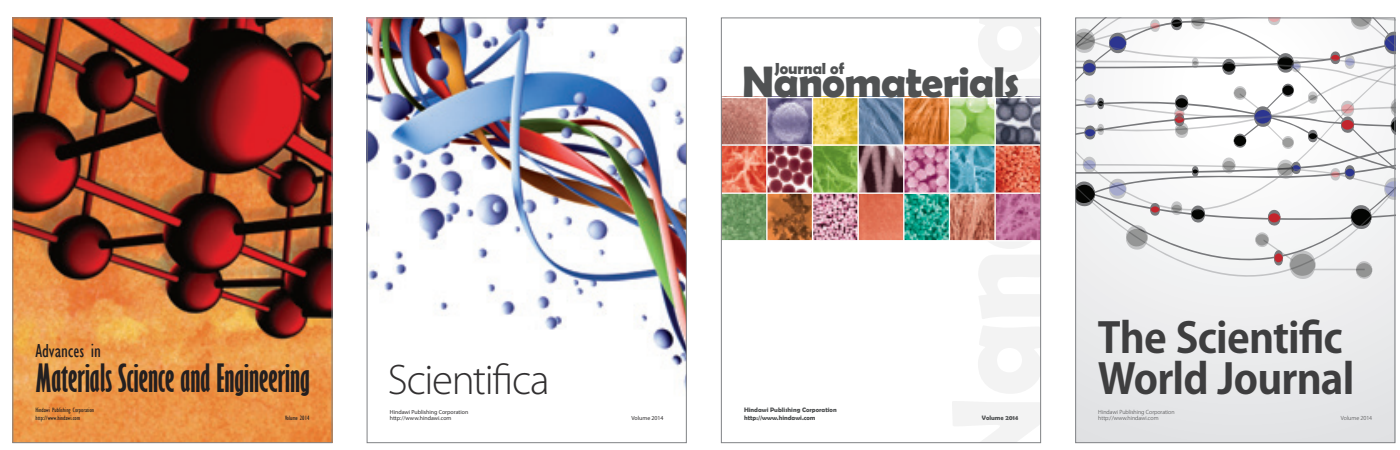

\section{The Scientific World Journal}
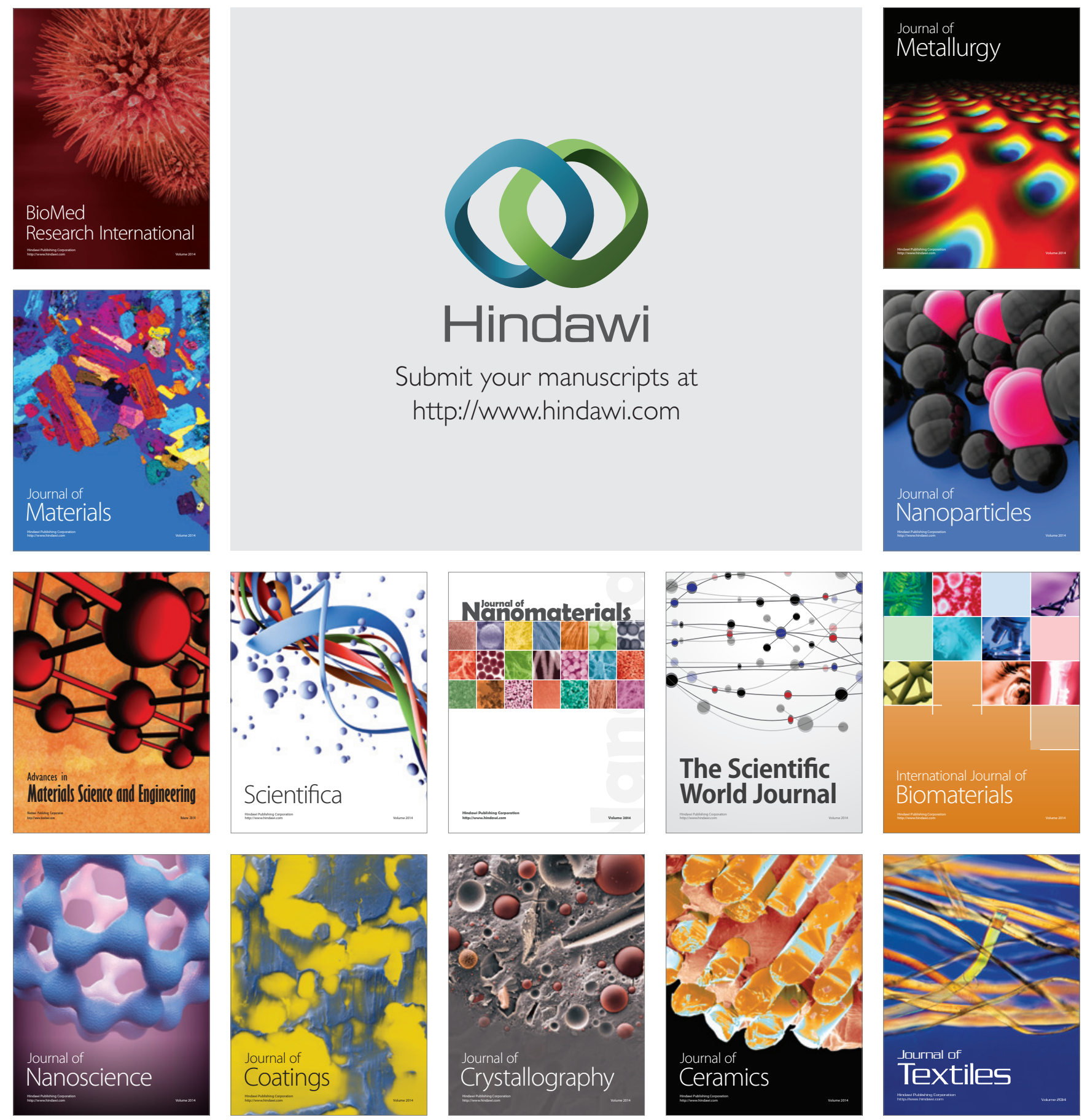